\title{
Situación Digital para Instituciones de Educación Superior: Modelo y Herramienta
}

\author{
Diego Samir Melo-Solarte ${ }^{(1) \star}$, Paula Andrea Díaz ${ }^{(2,3)}$, Omar Antonio Vega ${ }^{(1)}$ y Ciro Alfonso Serna ${ }^{(4)}$ \\ (1) Univ. de Manizales, Fac. Ciencias e Ingeniería, Cra 9 Nro 19-03, Manizales - Colombia \\ (2) Univ. de Manizales, Fac. Ciencias Sociales y Humanas, Cra 9 Nro 19-03, Manizales - Colombia \\ (3) Centro de Educación a Distancia (CEDUM), Cra 9 Nro 19-03, Manizales - Colombia \\ (4) Univ. de Manizales, Fac. Ciencias Contables y Administrativas, Cra 9 Nro 19-03, Manizales - Colombia \\ (e-mail: mdiego@umanizales.edu.co; pauladg@umanizales.edu.co; oavega@umanizales.edu.co; \\ redesomciro@hotmail.com)
}

${ }^{*}$ Autor a quien debe ser enviada la correspondencia

Recibido Mar. 27, 2018; Aceptado May. 29, 2018; Versión final Jun. 12, 2018, Publicado Dic. 2018

\section{Resumen}

Se propone un modelo para medir la situación digital en instituciones de educación superior. El modelo tiene un componente conceptual que define su estructura, un componente procedimental que determina su aplicación y una herramienta de software que articula los anteriores componentes para facilitar la operacionalización del mismo. Adicionalmente, se describe un estudio de caso desarrollado en una institución universitaria colombiana para evaluar el modelo considerando su efectividad, sus debilidades y su alcance. Entre los principales resultados obtenidos, está la validez del modelo para identificar el índice de situación digital para todos los integrantes de una comunidad universitaria, encontrando que las competencias axiológicas y productivas son las de menor valoración. Se concluye que toda institución debe contar con planes de gestión tecnológica que determine la viabilidad de la adquisición de tecnologías. Además, debe contar con estrategias de apropiación para reducir brechas y lograr mejor aprovechamiento de la inversión. digital

\section{Digital Situation for Higher Education Institutions: Model and Tool}

\begin{abstract}
This paper presents the proposal of a model to measure the digital situation in higher education institutions. The model is composed of a conceptual component that defines its structure, a procedural component that determines its application in an institution and a software tool that articulates the previous components in order to facilitate its functioning. In addition, this article describes a case study developed in a Colombian university institution for evaluating the model considering its effectiveness, its weaknesses and its scope. Among the main results obtained, there is the validity of the model to identify the digital situation index for all the members of a university community, finding that the axiological and productive competences are the least valued. It is concluded that the institution must have technological management plans that determine the viability of the acquisition of technologies. Also, it must have appropriation strategies to reduce gaps and achieve better use of the investment.
\end{abstract}

Keywords: e-learning; digital inclusion; digital divide; digital inclusion index; technological literacy; digital situation 


\section{INTRODUCCIÓN}

Este artículo presenta la propuesta de un modelo que permite identificar y analizar la situación digital en Instituciones de Educación Superior (IES), adicionalmente plantea el procedimiento base para la implementación del modelo, guiado por algunas recomendaciones logradas por la experiencia del equipo investigador, estos elementos se articulan en una herramienta de software, creada especialmente para facilitar la implementación del modelo y obtener unos resultados preliminares para las personas que hacen parte del proceso evaluativo. Es claro que en la actualidad, las tecnologías han permeado la sociedad y sus organizaciones, a la vez que se han convertido en elementos fundamentales para contribuir en el desarrollo y en el crecimiento, aunque también es claro que traen consigo problemáticas que afectan de alguna manera al ser humano: contaminación por desechos tecnológicos, alteración de los hábitos de las personas, problemas de orden sicológico, entre otras; razón por lo cual han surgido investigaciones que buscan abordar diferentes fenómenos desencadenados por la relación entre tecnologías y sociedad (Vallejo y Patiño, 2014). Es así que esta investigación aborda la problemática del acceso, uso y aprovechamiento tecnológico en las comunidades universitarias, quienes se han visto beneficiadas por la implementación de las Tecnologías de la Información y Comunicación (TIC) en diferentes procesos académicos y/o administrativos y también en el ejercicio diario de las personas que hacen parte de estas comunidades. El incremento masivo de TIC en el ámbito universitario, ha llevado a las IES a pensar, reflexionar y crear estrategias que permitan optimizar y aprovechar mejor los recursos que están a su disposición, pues es evidente, que en muchas ocasiones carecen de una perspectiva clara que indique la intencionalidad para obtener una determinada tecnología, o incluso, se desconoce el estado actual de la institución en el área, lo cual incide directamente en la proyección y planeación de una estrategia de aprovechamiento tecnológico que permita evaluar con claridad la necesidad, el uso y su impacto.

Existen modelos para la gestión tecnológica en las organizaciones, ejemplo de ello son: Cobit, cuyo modelo se centra en el planteamiento de mecanismos para evaluar y monitorear los sistemas de información y las tecnologías de una empresa (Isaca, 2012); Val it, cuyo modelo que se caracteriza por plantear mecanismos que permiten determinar el valor de negocio asociado a las inversiones tecnológicas que realiza una determina empresa (Poorebrahimi et al., 2016); podemos decir que estos modelos se centran en la gobernabilidad de las tecnologías que tiene un organización, desde su adquisición hasta ser desechadas, pasando por diferentes niveles de uso, obsolescencia y actualización; sin embargo, ninguno de ellos permite evaluar y medir el nivel de conocimiento y apropiación que tiene el usuario final sobre las diferentes tecnologías que están a su disposición, factor que se torna determinante a la hora de evaluar la pertinencia e impacto que puede tener una tecnología dentro de una organización. Por otra parte, es importante resaltar que se parte de la premisa que plantea que las IES tienes una responsabilidad directa con la sociedad y son las llamadas a impactarla (Naval y Ruiz, 2012), podemos considerar a las universidades como pequeños micro-mundos de exploración, donde todo lo que ocurre a su interior termina trascendiendo parcial o totalmente a la sociedad; por ende, no es extraño afirmar que el uso que se les da a las tecnologías dentro de la Universidad, será el mismo que se da por fuera de ella, al menos en el caso de los estudiantes que recién egresan de una IES. De esta manera, la pretensión de este trabajo se enfoca en identificar la situación digital de las IES para así contribuir en el diseño de estrategias que faciliten la apropiación tecnológica y así poder llegar a impactar más y de mejor manera a la sociedad.

La adquisición de tecnologías no puede ser un asunto de actualidad o "moda", o solamente de renovación por obsolescencia, las inversiones en tecnología deben ir de la mano con las necesidades reales y la proyección esperada; es claro que las organizaciones tienen que hacer grandes inversiones económicas para mantener actualizadas sus tecnologías, sin embargo, cabe preguntarse: ¿está el personal preparado para usar una nueva tecnología?, ¿es realmente necesario adquirir una nueva tecnología? o lo que es peor aún, ¿la relación costo/beneficio es significativa y se logra realmente obtener provecho de la nueva inversión? Evaluar la situación real de una organización frente al uso y apropiación tecnológica, es un punto de partida importante a la hora de pensar en nueva inversión que involucre dispositivos actualizados, las IES no son ajenas a este propósito (Berrío y Rojas, 2014); si bien, existe la convicción de renovar la infraestructura y estar a la vanguardia, vale la pena analizar el nivel de conocimiento y aprovechamiento que tiene una comunidad universitaria sobre los dispositivos tecnológicos que tiene a su disposición, esto permitiría hacer una inversión más consecuente con las necesidades, a la vez que permite construir planes y estrategias de inmersión tecnológica, coherentes y efectivos; la idea de construir un modelo que permita identificar la situación digital en las IES, busca recabar en la comunidad académica (estudiantes, docentes y administrativos), para determinar qué tanto una persona conoce, usa y aprovecha las tecnologías y de esta manera también es posible conocer el estado de la institución en este campo.

\section{REFERENTES TEÓRICOS}

Con el fin de caracterizar los pilares teóricos que dan forma a esta investigación y unificar el concepto base de este trabajo, se realizó un recorrido por los principales autores en el área, a continuación se esboza la 
definición de inclusión digital y sus términos asociados, adicional a ello, se presentan algunos trabajos relevantes en este campo. El auge y la penetración de las tecnologías en diferentes ámbitos de la sociedad, paulatinamente trajeron consigo diferentes problemáticas, especialmente causadas por la diferencia de posibilidades que puede tener una persona que dispone de las TIC y otra que está excluida de ellas. EI Consejo Europeo fue una de las primeras instituciones que comenzó a utiliza el término Inclusión Digital (eInclusion), cuando se aprueba el plan de acción e-Europe, el cual estaba orientado a la construcción de políticas para no dejar a ningún grupo social, aislado de lo que se denominó como "revolución digital" (Echeverría y Unceta, 2012). A partir de este momento, con la construcción de algunas definiciones que buscan clarificar el término o ajustarlo a ciertas características sociales.

Es claro que el término inclusión digital no solo se reduce a ser interpretado como el acceso a recursos computacionales y medios de comunicación, sino a la cualificación de las personas para lograr un uso efectivo de los recursos tecnológicos como lo afirma (Bellini et al., 2016).También es claro que las tecnologías por si solas no tienen ningún grado de relevancia, se necesita de la apropiación tecnológica a fin de poder sacar provecho del conocimiento logrado frente al uso e impacto de las tecnologías, siendo así "no se pude hablar solamente que la inclusión digital requiere disponibilidad del recurso y alfabetización digital, sino que se requiere estrategias de apropiación tecnológica" (Vega y Rodríguez-Baena, 2008). Las herramientas tecnológicas se han ido incorporando paulatinamente a las actividad universitarias dirigidas por los docentes, la percepción que los docentes tienen del rol de las TIC y de su importancia inciden en la apropiación de dichas herramientas (Tapasco y Giraldo, 2017) y en el impacto que se pueda llegar a tener.

Una definición que se acerca al concepto manejado en este proyecto, es la planteada por (Vega y RodríguezBaena, 2008) quienes la consideran como "el conjunto de políticas y estrategias tendientes a eliminar los obstáculos que limitan o impiden la participación activa y el aprovechamiento de las TIC en la economía y en la sociedad de la información, sin exclusión alguna", lo cual a su vez señala que la inclusión digital es un movimiento de carácter social, que supera la donación de equipos de cómputo o la baja de tarifas de los mismos, cuyo objetivo es tener acceso a las tecnologías y con su uso llegar a impactar la sociedad. Cuando se utiliza el término inclusión digital, también surgen expresiones relacionadas y que en muchas ocasiones son tomadas como sinónimos, tal vez el término que más se utiliza con esta característica es el de "brecha digital", haciendo alusión a la exclusión tecnológica de un sector de la población, dado en gran medida por el desconocimiento que la gente tiene sobre las tecnologías o la ausencia de ellas (Çoklar et al., 2017); a su vez, trae de la mano otro concepto asociado al conocimiento de las tecnologías y es el de "alfabetización digital", entendido como la competencia que tiene las personas para interactuar con las TIC (Guzmán-Simón et al., 2017), pero que igualmente se queda corto ya que el uso no implica aprovechamiento tecnológico, pero con él, se apunta a resolver la primera parte de la "brecha digital" que puede existir en la sociedad.

Brecha digital es probablemente es el concepto pionero en la reflexión sobre el impacto social de las tecnologías, esto dado por las evidentes diferencias de oportunidades en el desarrollo de las comunidades, brecha digital puede definirse como "la distancia tecnológica entre individuos, familias, empresas, grupos de interés, países y áreas geográficas en sus oportunidades en el acceso a la información y a las tecnologías de la comunicación y en el uso de Internet para un amplio rango de actividades" (Pedraza et al., 2012) la introducción de las TIC en diferentes ámbitos vinculantes con las personas, trae consigo desajustes sociales, que requieren de estrategias de penetración pero a la vez empoderamiento para reducir las brechas creadas por la tecnología (Várallyai et. al., 2015). Lo anterior señala una evolución conceptual al considerar la brecha digital desde el acceso, pasando por el uso, y llegando a un nivel de apropiación tecnológica, este último aspecto demarca el valor agregado o el beneficio que puede obtener una persona, a partir del uso consciente de las TIC; en otras palabras, hablar de brecha digital no solo es manifestar inconvenientes de acceso a la tecnología, sino también hablar de una problemática que genera disparidad en aspectos económicos, educativos, cognitivos, entre otros, originados en gran medida por la posibilidad de crear oportunidades con el uso de las tecnologías, para aquellos que las conocen y disponen de ellas (Vega y Rodríguez-Baena, 2008).

Es claro que hoy en día, el uso de las tecnologías de la información y comunicación ha aumentado considerablemente, tema que no es ajeno a las comunidades universitarias, donde los diferentes actores: estudiantes, docentes y administrativos, cada día más se ven abocados a apoyarse en las tecnologías para desempeñar sus labores; para el caso de los estudiantes que se los puede catalogar como "nativos digitales" y que están a la vanguardia con los avances tecnológicos, pero sin lograr un aprovechamiento significativo (Thompson, 2013); y por otra parte los docentes y administrativos, catalogados como "migrantes digitales" que requieren de un proceso de formación y adaptación al uso de las tecnologías por demanda del mercado y de su campo laboral (Hakkarainen et al., 2015), (Rush y Renguette, 2017).

Para lograr una apropiación efectiva de las tecnologías en el ámbito universitario, se requiere que los actores de las IES tengan actitudes favorables a las TIC y que las instituciones se comprometan con el proceso de alfabetización digital en su comunidad académica como lo manifiesta (Romero et al., 2015) quien 
adicionalmente muestra en su estudio, que esta es una manera de confrontar a los docentes contra lo que para muchos es desconocido, añadiendo que los profesores universitarios mayores de 50 años son los que menos usan las TIC y donde se requiere aplicar un plan estratégico para que ellos puedan incorporarlas dentro de sus actividades cotidianas o académicas; para varias universidades se hace imperativo crear estrategias que incentiven la incorporación de gradual y efectiva de las tecnologías (Lagunes-Dominguez, et al., 2015).

En su documento las TIC en el aula: percepciones de los profesores universitarios (Riascos-Erazo, et al., 2010), buscan determinar las apreciaciones de los profesores frente al uso de tecnologías dentro del aula de clase tanto para universidades públicas como privadas, este procedimiento lo realizan a través de encuestas y posteriormente su análisis a través de herramientas especializadas; entre su principal conclusión está en que la universidad privada el uso de las TIC en el aula de clase es alta, ya que en estas instituciones es un elemento clave del quehacer académico, mientras en las universidades públicas es medio evidenciando que la institución no muestra interés en su cualificación docente para aprovechar mejor las tecnologías. Sin embargo para (Drossel, et al., 2017) la brecha digital entre IES públicas o privadas, no está causada por la disponibilidad de tecnología, sino por una adecuada formación de su personal y la colaboración entre ellos, para lograr desarrollar competencias concernientes al uso adecuado de las TIC; (Romero et al., 2015) posiciona a las tecnologías como un elemento sustantivo de la concepción pedagógico contemporánea, dando a entender que las tecnologías deben estar implícitas en el aula de clase y que la formación o capacitación del docente es fundamental en pro del aprovechamiento de dichos recursos, Para lograr la articulación entre el uso de las tecnologías y las temáticas disciplinares.

En gran medida la inclusión de tecnológica en el aula de clase, es motivadas directamente por las habilidades, que autónomamente han desarrollado los estudiantes, siendo esta una de las razones por lo cual los docentes no se pueden quedar rezagados y terminan incorporando las TIC en sus procesos, sin embargo, existe la imperiosa necesidad de contar con un plan estratégico de alfabetización digital (Capilla et al., 2015), el cual conduzca a un mejor aprovechamiento tecnológico dentro del ámbito universitario, centrándose principalmente en grupos de usuarios y sus características, dejando en un segundo plano los procesos en los que son usadas las tecnologías. (Ferro et al., 2011). En la investigación realizada por (Vallejo y Patiño, 2014), se puede encontrar con la propuesta de algunos instrumentos para evaluar el nivel de apropiación tecnológica en instituciones educativas, adicional a ello, (Corona et. al., 2017), plantean una metodología para lograr la apropiación tecnológica en las instituciones educativas, además propone un modelo para determinar el impacto de las tecnologías en procesos de enseñanza y aprendizaje; en el mismo sentido se ha encontrado investigaciones como las de (Várallyai et. al., 2015), que plantea una propuesta metodológica para medir la brecha digital en la población de un país, integrando diferentes variables como: los niveles educativos y económicos de la población, los géneros, las edades, las características de la vivienda y aspectos culturales entre otros.

Conocer el estado digital de una organización o de un país, se constituye en el primer paso para identificar estrategias o mecanismos que contribuyan en el crecimiento y en el desarrollo socioeconómico (SerranoSantoyo y Rojas-Mendizabal, 2017), frente a ello se han visto varias investigaciones que se preocupan por construir un esquema válido y apropiado, que permita evaluar no solo aspectos asociados a la disponibilidad de recursos tecnológicos, sino también el conocimiento, el uso, la apropiación, el impacto, los residuos generados, entre otros; en el campo educativo, investigaciones como: (Berrío y Rojas, 2014), (Vallejo y Patiño, 2014), (Almerich et al., 2016), (Ruiz, 2016), (Corona et al., 2017), entre otros; han realizado diferentes planteamientos y propuestas concernientes a evaluar la situación digital de las IES con miras a que estas instituciones logren un mayor beneficio por el uso de las TIC en sus diferentes proceso; sin embargo, estas investigaciones han considerado especialmente a dos actores las comunidades universitarias: docentes y estudiantes; dejando de lado la parte administrativa de las universidades.

Uno de los referentes más usados a la hora de definir un esquema para clasificar las competencias que debe tener una persona a la hora de medir la situación digital en una IES, es el plateado especialmente por (Unesco, 2008), donde se consideran cinco dimensiones de análisis, con el fin de determinar el nivel de una competencia o del conocimiento digital en el que se encuentran las personas: (i) Tecnológica, se refiere a los conocimientos básicos sobre el funcionamiento de las tecnologías y conocimiento sobre programas de ofimática (procesador de texto, hojas de cálculo y programas de presentación); (ii) Informacional, con esta dimensión se busca identificar los conocimientos y habilidades que tienen las personas para la selección, búsqueda y tratamiento de la información a través de medios tecnológicos; (iii) Axiológica, entendida como los valores y principios que determinan el uso socialmente correcto de las tecnologías, con beneficio personal o grupal; (iv) Pedagógica, conocimiento sobre las implicaciones del uso de las TIC indicando sus posibilidades y su alcance, orientando esta dimensión a las capacidades para darlas a conocer y enseñar su exploración, a la vez que también se las usa como mediaciones para compartir otros conocimientos; y (v) Comunicativa, capacidades para establecer y mantener contactos con los demás, con propósitos lúdicos, comerciales o académicos. 
Otros estudios, como el de (Saavedra y Rodríguez, 2013), plantean modificaciones en el esquema, incorporando dos nuevas dimensiones para evaluar competencias de gestión e investigación a través del uso de las TIC, y eliminando las dimensiones axiológica e informacional; por otra parte, (Vera et al., 2014) crea un mecanismo para evaluar las competencias de los docentes, a partir de adaptaciones hechas a las dimensiones planteadas por (Unesco, 2008), en su propuesta se establecen cuatro dimensiones: la primera enfocada en las características del sujeto, la segunda el interés, la tercera el conocimiento y la cuarta la aplicación en el campo educativo; (Paz et al., 2013) hacen uso de las dimensiones planteadas por la Unesco y le agregan una quinta denominada: Desarrollo profesional la cual es entendida como el campo de acción de las tecnologías para una persona que egresa del mundo universitario e ingresa al campo laboral.

Varias investigaciones usan las dimensiones planteadas por la Unesco en el 2008, para evaluar las competencias digitales en docentes o en estudiantes, haciendo adaptaciones según el tipo de organización o el foco investigativo; para el caso de esta investigación se plantea analizar la situación digital de una IES, usando de base la clasificación de competencias planteada por la Unesco, pero ampliando su espectro a todos los actores de una comunidad universitaria: docentes, estudiantes y administrativos, además, focalizando el análisis en la apropiación y el aprovechamiento tecnológico dentro del rol que cada uno desempeña.

\section{METODOLOGÍA}

Para el desarrollo de este proyecto se toma como referente base el marco conceptual asociado a brecha e inclusión digital, posibilitando la construcción de un modelo que aborde el estudio de las competencias tecnológicas dentro de una comunidad universitaria, para ello este proyecto plantea la ejecución de cinco fases:

Fase 1: en la fase inicial de este proyecto, se hace un análisis de literatura relacionada con brecha digital, estrategias de inclusión digital, alfabetización tecnológica y mecanismos de medición de situación digital, esta etapa conceptualiza y caracteriza los principales elementos que hacen parte de los mecanismos para medir el conocimiento y aprovechamiento tecnológico en una IES;

Fase 2: en esta fase se procede a diseñar, conceptualizar y construir un modelo que permita identificar las habilidades tecnológicas que tienen las personas que hacen parte de una comunidad universitaria, contrastando dichas competencias con sus actividades diarias y las necesidades tecnológicas que ellas demandan (Ruiz, 2016), la pretensión del modelo es medir el nivel en que se encuentran cada persona, grupo e incluso una institución frente al conocimiento y apropiación tecnológica, en aras de lograr dentro del ejercicio académico y administrativo, cada vez más, un mayor provecho a las TIC;

Fase 3: para esta fase, se operacionaliza el modelo, con la construcción de los instrumentos que permiten identificar y medir las competencias tecnológicas, que poseen los diferentes personas que hacen parte de una comunidad universitaria dentro del ejercicio de cada uno de sus roles (estudiante, docente y administrativo);

Fase 4: En esta fase el equipo investigador realizó el análisis, diseño e implementación de una aplicación que responda a los requerimientos del modelo planteado, a los instrumentos que hacen parte de él y que a su vez facilite la interacción con la comunidad académica, viabilizando la recolección de los datos en línea y entregando para cada actor evaluado un resumen de los resultados obtenidos, con la posibilidad de compararse con la media correspondiente a su rol. Fase 5: en la última fase de este proyecto, se implementa un estudio de caso en una institución universitaria, lo cual busca analizar la situación digital de la institución a la vez que se evalúa la validez de los instrumentos y la efectividad del modelo planteado; finalmente, son analizados y presentados los principales resultados obtenidos.

\section{MODELO DE MEDICIÓN DE LA SITUACIÓN DIGITAL EN IES}

El modelo teórico-procedimental propuesto en esta investigación, busca medir los niveles de conocimiento, uso y aprovechamiento de recursos tecnológicos en instituciones de educación superior, la propuesta ha sido construida en el marco del proyecto de investigación denominado: "Modelo de medición de situación digital en instituciones de educación superior en Colombia." el cual tiene por objetivo, caracterizar y evaluar las competencias tecnológicas que tienen los diferentes actores que hacen parte de una comunidad académica universitaria.

En la primera parte de esta investigación se analizaron diferentes propuestas orientadas a medir el estado digital en el que se encuentran las IES, en esta instancia, se identificó que uno de los principales referentes usados para determinar qué competencias o habilidades se debe evaluar en un ambiente universitario, es el 
planteado por (Unesco, 2008) , quien propone cinco dimensiones a ser evaluadas: 1) Tecnológica, orientada a identificar los mecanismos y el lugar de acceso a recursos tecnológicos; 2) Informacional, evalúa la capacidad de búsqueda básica de contenidos temáticos; 3) Pedagógica, cuya finalidad se enfoca en evaluar el uso de las tecnologías para procesos educativos; 4) Comunicacional, evalúa el uso de las tecnologías con fines de contacto y transmisión de datos con otros; 5) Axiológica, que evalúa el uso ético de las tecnologías. Sin embargo, es crucial para esta investigación identificar los factores que determinan el aprovechamiento de las TIC, para lo cual se ha planteado una sexta dimensión de orden productivo, con ella se pretende identificar la trascendencia que llegan a tener las tecnologías tanto en el proceso formativo de las personas, como en sus actividades cotidiana, es decir, cuando las personas usan las tecnologías con el ánimo de mejorar una determinada labor o sacando provecho de ellas.

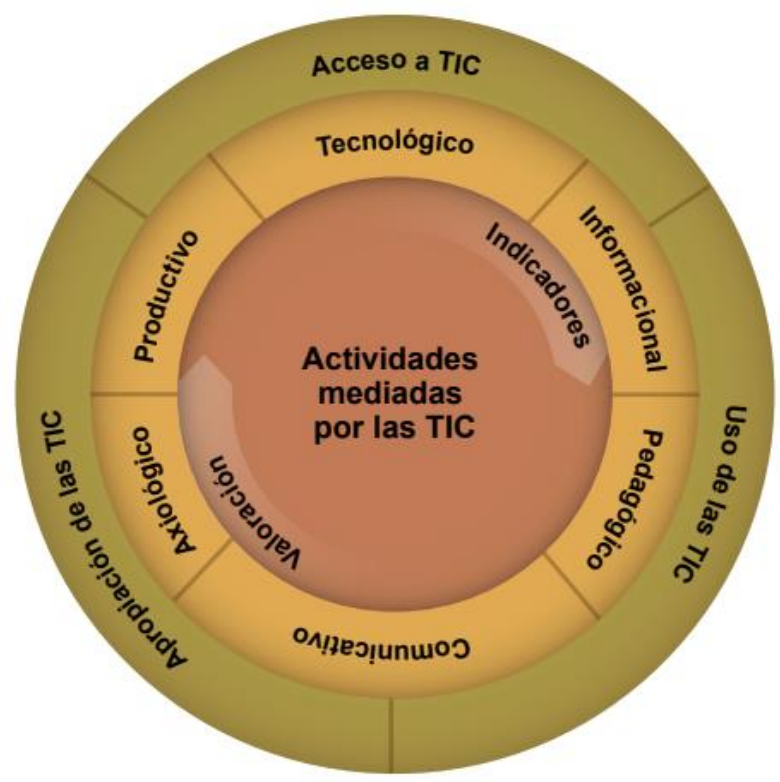

Fig. 1: Modelo para Identificar la Situación Digital en Instituciones de Educación Superior

La Fig. 1 Muestra la estructura general del modelo propuesto el cual se compone de tres niveles o capas: el núcleo del modelo está compuesto por el conjunto características que determinan las actividades mediadas por las TIC, sobre las cuales se quiere identificar el conocimiento o habilidad que tiene o debe tener cada persona que cumple un rol dentro de una comunidad universitaria. Las características permiten identificar atributos específicos como: el lugar de acceso, el tiempo de uso, los dispositivos, los fines, los aplicativos, entre otros; con ello se busca conocer los patrones de comportamiento que tiene cada persona en el momento de interactuar una tecnología; cada característica se compone de un conjunto de preguntas, que permiten indagar a una persona sobre diferentes aspectos asociados al conocimiento y al uso de las tecnologías, además, las preguntas dependen del rol que desempeña una persona en el ámbito universitario, a su vez, cada pregunta y cada características tienen asociado una ponderación que determina el grado de relevancia que ella tiene para el cumplimiento de un rol o para la institución.

Las características a su vez son agrupadas en seis dimensiones, las cuales determinan el nivel de las competencias que tienen las personas en una determinado campo de acción: 1) tecnológica, que refiere a los conocimientos básicos para manipulación de las TIC, 2) informacional, que indica un conocimiento mayor en procura de nueva información o protección de la existente; 3) pedagógica, orientada al uso de las tecnologías en procesos de enseñanza y aprendizaje, 4) comunicativa, enfocada en el uso TIC para procesos de transmisión de información ya sea académica, personal o laboral; 5) axiológica, orientada al uso ético y moral de las tecnologías, acorde a políticas institucionales y legales ; 6) productiva, implica evaluar la apropiación que tienen las personas sobre los recursos tecnológicos, para explotar sus posibilidades y crear nuevas oportunidades.

Finalmente, las dimensiones son agrupadas en estadios como se observa en la Tabla 1, los cuales permiten determinar la situación tecnológica de cada persona y confrontarla frente a la media de la comunidad universitaria, esto desde la perspectiva de: 1) acceso a las TIC, determina el conocimiento tecnológico básico que tienen las personas e identifica la manera cómo pueden acceder a ella; 2) uso de las tecnologías como parte de actividades cotidianas, pero sin implicar una postura reflexiva frente a que estas pueden mejorar su condición de vida; 3 ) apropiación, el cual busca reflejar el uso consciente que tienen las personas frente a las tecnologías, tratando de que ellas sean usadas para mejorar sus actividades o lograr cierto nivel de productividad con ellas. 
Tabla 1: Agrupación del modelo en estadios, dimensiones y características.

\begin{tabular}{|c|c|c|c|c|c|}
\hline Estadios & Ponderado & Dimensiones & Ponderado & Características & Ponderado \\
\hline \multirow{2}{*}{ Acceso a TIC } & \multirow{2}{*}{15} & \multirow{2}{*}{ Tecnológico } & \multirow{2}{*}{100} & Lugar de acceso & 30 \\
\hline & & & & Tipo de acceso & 70 \\
\hline \multirow{10}{*}{ Uso de las TIC } & \multirow{10}{*}{30} & \multirow{4}{*}{ Tecnológico } & \multirow{4}{*}{20} & Frecuencia de uso & 20 \\
\hline & & & & Tiempo de uso & 20 \\
\hline & & & & Manejo de herramientas ofimáticas & 40 \\
\hline & & & & Carga/descarga de documentos & 20 \\
\hline & & \multirow{2}{*}{ Informacional } & \multirow{2}{*}{25} & Navegación convencional & 40 \\
\hline & & & & Actividades lúdicas & 60 \\
\hline & & \multirow{2}{*}{ Pedagógico } & \multirow{2}{*}{30} & Actividades académicas & 50 \\
\hline & & & & Producción de documentos ofimáticos & 50 \\
\hline & & \multirow{2}{*}{ Comunicativo } & \multirow{2}{*}{25} & Comunicación convencional en línea & 60 \\
\hline & & & & Uso de redes sociales en línea & 40 \\
\hline \multirow{23}{*}{$\begin{array}{c}\text { Apropiación de las } \\
\text { TIC }\end{array}$} & \multirow{23}{*}{55} & \multirow{4}{*}{ Tecnológico } & \multirow{4}{*}{13} & Manejo de herramientas ofimáticas en línea & 30 \\
\hline & & & & Manejo de software especializado & 30 \\
\hline & & & & $\begin{array}{l}\text { Personalización de herramientas } \\
\text { tecnológicas }\end{array}$ & 20 \\
\hline & & & & Herramientas de interacción especializada & 20 \\
\hline & & \multirow{3}{*}{ Informacional } & \multirow{3}{*}{19} & Trabajo colaborativo en línea & 30 \\
\hline & & & & $\begin{array}{l}\text { Búsqueda y organización de información } \\
\text { científica }\end{array}$ & 35 \\
\hline & & & & Divulgación y difusión académica & 35 \\
\hline & & \multirow{4}{*}{ Pedagógico } & \multirow{4}{*}{27} & Cursos en ambientes virtuales & 25 \\
\hline & & & & Consultorías o asesorías en línea & 25 \\
\hline & & & & Actividades académicas en línea & 25 \\
\hline & & & & Sesiones académicas mediadas por TIC & 25 \\
\hline & & \multirow{3}{*}{ Comunicativo } & \multirow{3}{*}{19} & $\begin{array}{l}\text { Procesos de acompañamiento y } \\
\text { retroalimentación en línea }\end{array}$ & 25 \\
\hline & & & & $\begin{array}{l}\text { Notificaciones o alertas académicas y/o } \\
\text { profesionales en línea }\end{array}$ & 40 \\
\hline & & & & Opciones de comunicación con tecnologías & 35 \\
\hline & & \multirow{4}{*}{ Axiológico } & \multirow{4}{*}{9} & $\begin{array}{l}\text { Respeto y promoción de la propiedad } \\
\text { intelectual }\end{array}$ & 30 \\
\hline & & & & Actividades éticas y legales en línea & 20 \\
\hline & & & & $\begin{array}{l}\text { Promoción del uso consciente y racional de } \\
\text { las TIC }\end{array}$ & 25 \\
\hline & & & & Interés por la tecnología & 25 \\
\hline & & \multirow{5}{*}{ Productivo } & \multirow{5}{*}{13} & Comercio electrónico & 25 \\
\hline & & & & Participación ciudadana en línea & 10 \\
\hline & & & & Eventos académicos en línea & 15 \\
\hline & & & & Capacitación en línea & 25 \\
\hline & & & & $\begin{array}{l}\text { Incorporación de TIC en su renglón } \\
\text { productivo }\end{array}$ & 25 \\
\hline
\end{tabular}

Para la interacción con el personal evaluado, se ha creado un cuestionario, compuesto por una serie de preguntas, donde cada una está vinculadas a una característica y se evalúa de acuerdo a la escala creada bajo la estructura Likert (Frecuentemente / Esporádicamente / Nunca), cada elemento de la escala tiene un valor asignado (100 / 50 / 0). Por otra parte, como se muestra en la Tabla 1, las características, las dimensiones y los estadios, cuentan con una ponderación, lo cual permite medir para cada persona, el nivel en el que se encuentra sus competencias tecnológicas, la ponderación o valor asignado, depende de la percepción que tenga el equipo evaluador, respecto a la importancia o relevancia que tenga una competencia, la intencionalidad de la evaluación, el entorno, las necesidades, entre otras razones. Al ponderado obtenido 
después aplicar este modelo, se le ha denominado "índice de situación digital" y aunque es un valor numérico, está asociado a un conjunto de atributos que posibilitan el análisis cualitativo, los cuales a su vez permiten evaluar en detalle los aspectos y competencias tecnológicas de una persona o una comunidad, identificando sus fortalezas o debilidades; de esta manera, es posible dilucidar el estado tecnológico en el cual se encuentra una IES y cada una de las personas que hace parte de ella, con la intencionalidad que dicho resultado, conduzca a crear mecanismos que contribuyan en el uso efectivo y productivo de las TIC.

Ahora bien, para realizar el cálculo que determina el índice digital ya sea por estadio, dimensión o característica, se procede inicialmente respondiendo las preguntas que determinan esa característica como se muestra la Tabla 2, donde a manera de ejemplo, se visualiza una sección del modelo que representa las respuestas de un participante, de esta manera se tendría que las características: "Actividades académicas" se satisface en un $62.5 \%$ y "Producción de documentos ofimáticos" se satisface en un $33.3 \%$; por otra parte la dimensión "Pedagógica" se satisface en $47.9 \%$, como resultado de aplicar los porcentajes de cada característica al puntaje obtenido; así sucesivamente se escala para llegar a los estadios y también lograr el índice global.

Tabla 2: Ejemplo de una sección del modelo

\begin{tabular}{|c|c|c|c|c|c|c|c|c|}
\hline Estadio & $\%$ & Dimensión & $\%$ & Característica & $\%$ & Pregunta & Respuestas & $\begin{array}{l}\text { Puntuación } \\
\text { por } \\
\text { respuesta }\end{array}$ \\
\hline \multirow{7}{*}{$\begin{array}{l}\text { Uso de } \\
\text { las TIC }\end{array}$} & \multirow{7}{*}{30} & \multirow{7}{*}{ Pedagógico } & \multirow{7}{*}{30} & \multirow{4}{*}{$\begin{array}{l}\text { Actividades } \\
\text { académicas }\end{array}$} & \multirow{4}{*}{50} & $\begin{array}{l}\text { ¿Recibe trabajos } \\
\text { académicos por el correo } \\
\text { electrónico? }\end{array}$ & Frecuentemente & 100 \\
\hline & & & & & & $\begin{array}{l}\text { ¿Utiliza el correo } \\
\text { electrónico como medio de } \\
\text { comunicación con los } \\
\text { estudiantes? }\end{array}$ & Esporádicamente & 50 \\
\hline & & & & & & $\begin{array}{l}\text { ¿Utiliza el correo } \\
\text { electrónico como medio de } \\
\text { comunicación con otros } \\
\text { docentes? }\end{array}$ & Nunca & 0 \\
\hline & & & & & & $\begin{array}{l}\text { ¿Participa como asistente } \\
\text { en eventos académicos en } \\
\text { línea? }\end{array}$ & Frecuentemente & 100 \\
\hline & & & & \multirow{3}{*}{$\begin{array}{l}\text { Producción de } \\
\text { documentos } \\
\text { ofimáticos }\end{array}$} & \multirow{3}{*}{50} & $\begin{array}{l}\text { ¿Diseña y produce material } \\
\text { relativo a sus funciones } \\
\text { utilizando herramientas } \\
\text { informáticas? }\end{array}$ & Nunca & 0 \\
\hline & & & & & & $\begin{array}{l}\text { ¿En sus sesiones laborales } \\
\text { utiliza presentaciones } \\
\text { realizadas en herramientas } \\
\text { locales o en línea? }\end{array}$ & Frecuentemente & 100 \\
\hline & & & & & & $\begin{array}{l}\text { ¿Construye plantillas o } \\
\text { formatos para aspectos } \\
\text { laborales? }\end{array}$ & Nunca & 0 \\
\hline
\end{tabular}

\section{Procedimiento para la aplicación del modelo}

Con el ánimo de que el proceso de evaluación de conocimientos y habilidades tecnológicas sea exitoso dentro de cualquier comunidad universitaria, fruto de las experiencias logradas, el equipo investigador ha dispuesto un conjunto de pasos y recomendaciones a tener en cuenta:

(i) Definir objetivo, la primera recomendación es tener claro el objeto de la evaluación, es decir, determinar por qué se hará la evaluación de competencias digitales y para que se usarán los resultados, esto con el ánimo de poder determinar el alcance de este proceso, dentro de las necesidades propias de una institución universitaria;

(ii) Determinar grupo poblacional, aunque el modelo contempla tres grupos poblaciones o roles: estudiantes, docentes y administrativos, es importante determinar si serán evaluados los tres o solo alguno de ellos, cabe recordar que cada grupo debe ser abordado de forma diferente con el ánimo de contextualizar y eliminar temores del proceso o de la información que cada uno de ellos suministre, pues muchos suponen erróneamente que este tipo de evaluaciones serán usados para tomar represalias administrativas

(iii) Ajustar los instrumentos, por cada rol a evaluar existe un instrumento el cual está compuesto por un 
conjunto de preguntas y posibles respuestas, cada una de las preguntas tiene asignado una ponderación; es importante determinar la pertinencia de cada pregunta y validar si la ponderación está acorde a las características propias de la institución, pues se evidencia que en algunas organizaciones se prioriza algunos factores más que otros, o incluso puede ser que una institución solo desee aplicar sólo una parte de los instrumentos;

(iv) Recolección de datos, en esta etapa se aplica los instrumentos a la población objetivo, es importante contar con el apoyo de las directivas de la institución, para que el personal esté comprometido con el proceso y en dar respuesta a los cuestionarios, aún más conociendo que este proceso tomará aproximadamente 30 minutos; adicionalmente es importante, realizar una sensibilización del proceso y una socialización de los resultados sin individualizar los mismos; pues al presentar los resultados de forma individual pueden crearse escenarios de zozobra o discriminación;

(v) Analizar y evaluar resultados, una vez finalizado el proceso de recolección de datos, se inicia el proceso de evaluación de resultados, la herramienta permite la creación de reportes grupales o individuales por característica, dimensión o estadio; sin embargo, para analizar a profundidad el comportamiento de los ítems en consideración, se hace necesario otro tipo de herramientas de análisis estadístico avanzado y mecanismos para interpretación de datos cualitativos; y

(vi) Publicar los resultados, una vez terminado el proceso y determinado el índice de situación digital para una institución, es importante que estos sean conocidos por las directivas, con el ánimo de construir conclusiones conjuntas y diseñar planes o estrategias de apropiación tecnológica que permitan reducir brechas tecnológicas o mejorar el uso de las TIC en procurando una mayor productividad.

\section{ESTUDIO DE CASO}

Como mecanismo para poner a prueba, evaluar y validar el modelo propuesto en esta investigación, se diseñó un estudio de caso el cual tenía por objetivo analizar la situación digital de una comunidad universitaria, considerando los diferentes roles que hacen parte de ella: docentes, estudiantes y administrativos. Para poner en práctica este estudio de caso, inicialmente se instanció el software con los instrumentos a ser aplicados, con la intención de evaluar a los diferentes miembros de una comunidad universitaria; posteriormente se hizo un test de validación de los instrumentos con la finalidad de garantizar su coherencia y sus ponderados; una vez terminada esta labor se procedió a aplicar los instrumentos, para lo cual se envió el enlace del aplicativo a toda la comunidad universitaria, finalizado el proceso de recolección de datos, se procedió a evaluar la manera como respondió el aplicativo, se evalúo la eficiencia y pertinencia de los instrumentos, finalmente, se analizó los resultados de acuerdo con los datos obtenidos.

El aplicativo se convirtió en un gran aliado para realizar el levantamiento de datos en un corto tiempo, para poder instanciar y condensar todos los instrumentos, a la vez que permitió almacenar el resultado de la encuestas realizadas, logrando una participación del 9\% de la comunidad académica; por otra parte, el aplicativo permitió tener algunos informes de forma inmediata posibilitando a cada persona encuestada, que pudiera ver sus resultados y compararlos con sus compañeros. Respecto a los instrumentos, se pudo evidenciar que están compuestos por cuestionarios muy extensos, lo cual se tradujo en que el $2 \%$ de las personas que iniciaban el procedimiento para dar respuesta a la encuesta, a la mitad del mismo desistían; por otra parte, la forma como están compuestos los instrumentos y la posibilidad de asignar un valor a cada uno de los elementos que lo componen, permitió evaluar la situación digital en diferentes niveles, desde la obtención de un índice a nivel general, hasta conocer la ponderación ya sea por estadio, dimensión o característica; esto permite analizar los factores en estudio de este campo, desde la raíz misma donde se pueden presentar los inconvenientes, que se puede traducir a su vez, en estrategias de inclusión.

Cabe aclarar que el modelo está compuesto por tres instrumentos, estos a su vez se componen de una serie de preguntas con su respectivo valor, sin embargo, los instrumentos puede ser adaptado según necesidades del equipo que analiza la situación digital de una institución, ya sea aumentando o disminuyendo preguntas o sus valores. Frente a los resultados que se obtuvieron una vez aplicados los instrumentos, de puede destacar lo siguiente: en primera instancia se evidenció que un $42 \%$ de los empleados (docentes y administrativos) optó por responder los instrumentos de manera anónima, mientras que el $18 \%$ de los estudiantes se inclinó por esta opción; esto demuestra el temor de los empleados a dar respuesta sincera sobre sus conocimientos y habilidades, con el supuesto de que si no manifiestan el dominio del tema, podrían existir medidas contra ellos; por otra parte los estudiantes respondieron sin prejuicios a ser juzgados.

Conocedores de la extensión de los instrumentos, el equipo investigador trato de desarrollar una estrategia de motivación que lograra captar el mayor número de instrumentos respondidos, para ello se creó una interfaz de fácil y rápida interacción, donde se navega por páginas y cada página tiene una matriz de doce preguntas 
dispuestas a manera de cartas; cuando una persona responde la pregunta, automáticamente la carta se gira e inhabilita nuevas respuestas, con las cartas giradas se posibilitó el juego concéntrese el cual se usó para generar una puntuación.

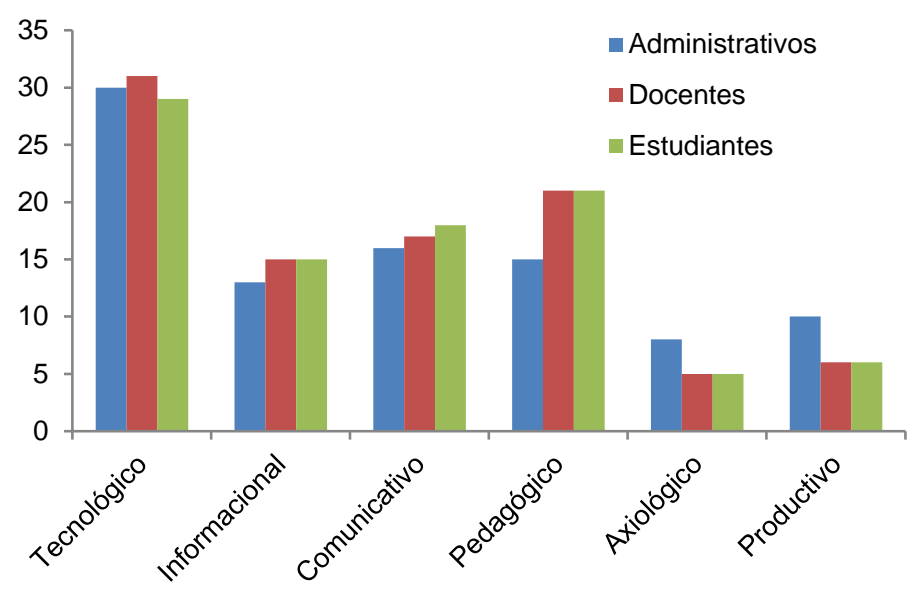

Fig. 2: Resultados por dimensiones

En la Fig. 2 se muestran los resultados por dimensiones y roles, obtenidos a partir del estudio de caso, en ello se encontró que la dimensión de mayor puntuación fue la tecnológica, lo cual demuestra que la mayoría de las personas tiene acceso a las tecnologías ya sea en su hogar, en el trabajo, en la institución educativa o incluso acceso a través de dispositivos móviles; sin embargo, se nota que los estudiantes tienen una pequeña variación a la baja, en gran medida producida porque ellos no cuentan con una autonomía económica.

En la dimensión Informacional se pudo evidenciar que quienes hacen mayor uso de las tecnologías para buscar nueva información o transmitirla son los docentes y estudiantes; en la dimensión comunicativa se evidencia que son los estudiantes quienes usan más las tecnologías con un alto propósito de comunicación lúdica a bajo costo, seguida de la comunicación para actividades educativas; en la dimensión pedagógica, se visualiza que tanto estudiantes como docentes son los que se benefician en gran medida de las tecnologías para apoyar sus procesos formativos, se focaliza mucho en la búsqueda, manipulación y almacenamiento de información propia de sus programas académicos; para las dimensiones: axiológica y productiva se puede observar que son los administrativos quienes buscan enseñar a sus colegas sobre el uso adecuado y productivo de los recursos tecnológicos, sin descuidar los valores sociales o morales que ellos pueden tener implícitos, son los administrativos quienes enfocan la ayuda de las TIC en la realización de sus actividades laborales cotidianas, aunque sin evidencia de diversificación de las tecnologías para encontrar o desarrollar nuevas posibilidades.

Finalmente, como parte del proceso de estudio de caso, el equipo investigador encontró la necesidad de facilitar la interpretación de los resultados entregados por la aplicación del modelo en cualquier IES, por lo cual se crearon unas escalas: deficiente, regular, bueno o sobresaliente; de esta manera, cada elemento evaluado individualmente puede ser comparado con la media del grupo de acuerdo a su rol, a la vez que se ubica en una posición de la escala, con lo cual se destaca otros resultados relevantes, entre ellos, que se pudo identificar que existe una baja apropiación tecnológica, pues el común denominador en los actores que hacen parte de la comunidad universitaria, es la subutilización de tecnologías, pues reconocen que sus dispositivos tienen mayor potencial del que aprovechan; por una parte se evidenció que los estudiantes son quienes más adquieren y renuevan sus tecnologías, sin embargo, a pesar de ser tecnología de punta y de gama alta, la siguen utilizando de la misma manera como lo hacían con el dispositivo que desecharon; los docentes son más prudentes a la hora de adquirir tecnologías, evalúan primero sus necesidades, se inclinan por dispositivos de gamas media con un ciclo de vida que rondan los 5 años para portátiles y 3 años para celulares; los administrativos son más dados a usar infraestructura computacional de la institución, utilizándola principalmente para actividades ofimáticas o interacción con plataformas a través de web.

\section{DISCUSION FINAL}

El modelo planteado en esta investigación, permite identificar y medir de manera efectiva las competencias tecnológicas de una comunidad universitaria, con lo cual es factible determinar fortalezas y/o debilidades de en los estadios: acceso, uso y apropiación de las TIC, los cuales agrupan las dimensiones: tecnológica, informacional, comunicativo, pedagógico y axiológico planteadas por la (Unesco, 2008), más la que incorpora esta investigación y que es de orden productivo; esto su vez se convierte en base para la toma de decisiones 
frente a nueva incorporación tecnológica o la reducción de brechas con las tecnologías disponibles en una institución. Cabe resaltar que los instrumentos que hacen parte del modelo recibieron una valoración positiva por su estructura y por la posibilidad de ser ajustados según las características particulares de una institución, de esta manera es posible evaluar en detalle, una determinada característica o competencia, sin embargo, entre mayor sea el grado de detalle para una evaluación, mayor va ser el tamaño del instrumento, repercutiendo en el tiempo requerido para el proceso y en el número de personas que van hacer parte de él.

Es posible concluir que la preocupación de las instituciones universitarias, inicialmente ha estado marcada por brindar acceso a las tecnologías, logrando una mayor cobertura; sin embargo, se encontró una débil respuesta frente al uso ético y moral de las TIC, con un aprovechamiento tecnológico reducido que no permite innovar frente a nuevas posibilidades y menos impactar la sociedad. Con la aplicación del modelo en un estudio de caso se pudo evidenciar que las tecnologías en el ámbito universitario, tienen una fuerte tendencia al uso informativo y lúdico, un nivel medio en apoyo a actividades cotidianas académicas y una baja preocupación por lograr réditos a través de la explotación de las tecnologías con fines productivos, resultados similares a los expresados por (Berrío y Rojas, 2014).

La actualidad enfrenta a las Universidades a una imperiosa necesidad de dominar las tecnologías para lograr un mejor aprovechamiento y trascender a la sociedad, se supone que el cuerpo docente debería liderar estos procesos, pero al igual que (Ruiz, 2016), en este trabajo se encontró que los docentes no cuentan con un nivel de competencias equilibrado, es decir, la percepción del docente, es de dominio medio-alto sobre las tecnologías, pero en el aula de clases no hay reflejo de ello, entendiendo las tecnologías como una herramienta complementaria o extensión de los escenarios formativos.

\section{CONCLUSIONES}

De acuerdo al trabajo presentado y a los resultados obtenidos, se pueden plantear las siguientes conclusiones principales:

1.- El modelo para medir el índice digital de una comunidad universitaria que plantea esta investigación, fue efectivo y ágil a la hora de determinar e identificar el conocimiento y las competencias tecnológicas que tienen los actores de una institución educativa, pero a pesar de ello, se pudo evidenciar que docentes y administrativos se sienten temerosos de responder un instrumento que evalúe sus competencias.

2.- La mayoría del personal sobre los cuales se aplicó el caso de estudio, son conscientes del papel que juegan las TIC de cara a mejorar su desempeño académico o laboral, sin embargo, los resultados obtenidos muestran un bajo nivel de uso de las tecnologías en el orden productivo, y un alto nivel de uso en el orden recreativo; lo cual lleva a concluir que existe bajo aprovechamiento de las tecnologías y poca preocupación por descubrir nuevas posibilidades que mejoren sus condiciones de vida laboral o académica.

3.- La evaluación por estadios, demuestra que existe una facilidad de acceso a las tecnologías, especialmente al tener disponible dispositivos con conexión a internet; el uso se centra en actividades rutinarias con una evidente subutilización de sus potencialidades; finalmente, la apropiación permite identificar que la mayoría de las personas que hacen parte de las comunidades universitarias son usuarios pasivos, pues son pocos los que se empoderan de las tecnologías para innovar, explotarlas y obtener mayores beneficios de ellas.

\section{AGRADECIMIENTOS}

Los autores agradecen el apoyo de la $\mathrm{U}$. de Manizales a través del proyecto de investigación: "Modelo de medición de situación digital en instituciones de educación superior en Colombia", dentro de la convocatoria interna 2014-1.

\section{REFERENCIAS}

Almerich, G., N. Orellana, J. Suárez-Rodríguez e I. Díaz-García, Teachers' information and communication technology competences: A structural approach, Computers and Education, 100, 110-125 (2016)

Bellini, C.G.P., M.M. Isoni y otros dos autores, Self-efficacy and anxiety of digital natives in face of compulsory computermediated tasks: A study about digital capabilities and limitations, Computers in Human Behavior, 59, 49-57 (2016)

Berrío C. y H. Rojas, La brecha digital universitaria: la apropiación de las TIC en estudiantes de educación superior en Bogotá (Colombia), Comunicar: Revista Científica Iberoamericana de Comunicación y Educación, (43), 133-142 (2014)

Capilla, M.M., J.M.Torres y F.R. Sánchez, Percepciones acerca de la integración de las TIC en el proceso de enseñanzaaprendizaje de la universidad, Pixel-Bit: Revista de medios y educación, 46, 103-117 (2015) 
Çoklar, A.N., N.D. Yaman e I.K Yurdakul, Information literacy and digital nativity as determinants of online information search strategies, Computers in Human Behavior, 70, 1-9 (2017)

Corona, A.G., F. Martínez-Abad y M.J. Rodríguez-Conde, Evaluation of digital competence in teacher training, Doi: http://doi.org/10.1145/3144826.3145367, In ACM International Conference Proceeding Series, Part F1322, 20 (2017)

Drossel, K., B. Eickelmann y R. Schulz-Zander, Determinants of teachers' collaborative use of information and communications technology for teaching and learning: A European perspective, doi:http://doi.org/10.1177/1474904116655811, European Educational Research Journal, 16(6), 781-799 (2017)

Echeverría, J. y A. Unceta, Citizenship and Participation in the European Electronic Space, doi:10.3989/arbor.2012.756n4007, Arbor, 188 (756), 725-732 (2012)

Ferro, E., N.C. Helbig y J.R. Gil-Garcia, The role of IT literacy in defining digital divide policy needs, doi: http://doi.org/https://doi.org/10.1016/j.giq.2010.05.007, Government Information Quarterly, 28(1), 3-10 (2011)

Guzmán-Simón, F., E. García-Jiménez e I. López-Cobo, Undergraduate students' perspectives on digital competence and academic literacy in a Spanish University, Computers in Human Behavior, 74, 196-204 (2017)

Hakkarainen, K., L. Hietajärvi y otros tres autores, Sociodigital Revolution: Digital Natives vs Digital Immigrants. In J. D. B. T.-I. E. of the S. \& B. S. (Second E. Wright (Ed.), 918-923 (2015)

Isaca, Cobit 5 Un Marco de Negocio para el Gobierno y la Gestión de las TI de la Empresa, Isaca Framework (2012)

Lagunes-Domínguez, A., C.A. Torres-Gastelú, M.A. Flores-García y A. Rodríguez-Figueroa, Comparativo del uso de Tecnologías de la Información y Comunicación (TIC) por Profesores de Dos Universidades Públicas de México, Formación Universitaria, 8(2), 11-18 (2015)

Naval, C. y M. Ruiz, Aproximación a la responsabilidad social universitaria: la respuesta de la universidad a la sociedad, Bordón 64(3), 103-115 (2012)

Paz, M.A., P. Espinosa e I.G. Porlán, Competencias tecnológicas del profesorado en las universidades españolas, doi:http://doi.org/10.4438/1988-592X-RE-2011-361-140, 361, 13-6 (2013)

Pedraza, L.F., A. Danilo, O.J. Salcedo y J. Octavio, Brecha Digital por Estatus Socio-Económico en la Localidad de Ciudad Bolívar de Bogotá (Colombia), Información Tecnológica, 23(6), 63-72 (2012)

Poorebrahimi, A., F. Razavi y F.S. Razavi, Presenting VALIT Frameworks and Comparing between Them and Other Enterprise Architecture Framework, Int. J. Advanced Networking and Applications, 7(4), 2805-2809 (2016)

Riascos-Erazo, S., D. Quintero-Calvache y G. Ávila-Fajardo, Las TIC en el aula: percepciones de los profesores universitarios, Educación y Educadores, 12(3), (2010)

Romero, G.H., C.G.M Aparicio y M.D.C.N Torres, Inclusión de las TICS en el trabajo académico de los profesores universitarios, Revista Electrónica sobre Tecnología, Educación y Sociedad, 1(4), (2015)

Ruiz, A.B.M., El profesorado universitario y las TIC, Análisis de su competencia digital, Revista de la Facultad de Educación de Albacete, 31(1), 133-147 (2016)

Rush M. y C.C. Renguette, Technological Literacy: A Framework for Teaching Technical Communication Software Tools, doi: http://doi.org/10.1080/10572252.2017,1385998, Technical Communication Quarterly, 26(4), 395-411 (2017)

Saavedra, M.F.C. y G.G.C. Rodríguez, Competencias TIC Para el Desarrollo Profesional Docente. Colección Sistema Nacional de Innovación Educativa con uso de Nuevas Tecnologías. doi: http://doi.org/10.1017/CBO9781107415324.004, Ministerio de Educación Nacional (2013)

Serrano-Santoyo, A. y V. Rojas-Mendizabal, Exploring a Complexity Framework for Digital Inclusion Interventions, Procedia Computer Science, 121, 212-217 (2017)

Tapasco, O.A. y J.A. Giraldo, Estudio Comparativo sobre Percepción y uso de las TIC entre Profesores de Universidades Públicas y Privadas, Formación Universitaria, 10(2), 03-12 (2017)

Thompson, P., The digital natives as learners: Technology use patterns and approaches to learning, doi: http://doi.org/https://doi.org/10.1016/j.compedu.2012.12.022, Computers \& Education, 65, 12-33 (2013)

Unesco, Estándares Unesco de Competencias en TIC para Docentes, Organización de Las Naciones Unidas Para La Educación La Ciencia y La Cultura (UNESCO), 1-28 (2008)

Vallejo, M. y M.R. Patiño, Instrumentos para Evaluar Apropiación Tecnológica, Revista Q Tecnología Comunicación Educación, 9(17), 1-26 (2014)

Várallyai, L., M. Herdon y S. Botos, Statistical Analyses of Digital Divide Factors, http://doi.org/https://doi.org/10.1016/S22125671(15)00037-4, Procedia Economics and Finance, 19, 364-372 (2015)

Vega, O.A. y L. Rodríguez-Baena, La inclusión digital como motor de desarrollo. Una opción para la Colombia rural, Sociedad y Utopía, Revista de Ciencias Sociales, (32), 75-95 (2008)

Vera J.Á., L.E. Torres y E.E. Martínez, Evaluación de competencias básicas en TIC en docentes de educación superior en México. Pixel-Bit. Revista de Medios y Educación, (44), 143-155 (2014) 